\title{
LA EVALUACIÓN DE IMPACTO ARQUEOLÓGICO DE LA RED DE GASIFICACIÓN DE GALICIA: CONSIDERACIONES METODOLÓGICAS Y SÍNTESIS DE RESULTADOS
}

\author{
THE EVALUATION OF ARCHAEOLOGICAL IMPACT OF THE GALICIAN GAS PIPELINE: \\ METHODOLOGICAL CONSIDERATIONS AND SYNTHESIS OF RESULTS
}

\author{
JORGE MIGUEL AYÁN VILA (*) \\ XESÚS AMADO REINO (*)
}

\section{RESUMEN}

Este artículo tiene como finalidad primordial ofrecer una síntesis valorativa de los trabajos arqueológicos realizados por el Grupo de Investigación en Arqueología del Paisaje de la Universidad de Santiago de Compostela durante la Fase de Evaluación del Impacto Arqueológico de los diferentes tramos proyectados de la Red de Gasificación de Galicia entre los años 1991 y 1997. En este sentido se exponen los resultados en términos de conocimiento arqueológico, desarrollo metodológico y gestión patrimonial de un proyecto que se presenta como un ejemplo de adaptación de la Arqueología a las demandas impuestas por la Gestión del Patrimonio Arqueológico en la actualidad. A este respecto, pretendemos aportar con nuestro análisis un conjunto de consecuencias significativas de un caso práctico concreto que pueda servir de referencia en el debate mantenido en nuestros días sobre la relación entre la dimensión investigadora (la llamada Arqueología de Investigación) y la orientación patrimonial (la denominada Arqueología de Gestión) de la disciplina arqueológica actual.

\footnotetext{
ABSTRACT

This article presents a critical summary of the results of archaeological surveys carried out during the Evaluation

(*) Laboratorio de Arqueoloxía e Formas Culturais (Grupo de Investigación en Arqueoloxía da Paisaxe) Instituto de Investigacións Tecnolóxicas. Universidade de Santiago de Compostela. Dirección Postal: Edificio Monte da Condesa USC-Campus Sur. 15782 Santiago de Compostela. Correo electrónico: phxurxo@ usc.es phsusoa@usc.es

El artículo fue remitido en su versión final el 20-II-2001.
}

Stage of the Archaeological Impact of the Galician Gas Pipeline by the University of Santiago Research Unit on Landscape Archaeology between 1991 and 1997. As an example of research that shows Archaeology's response to the contemporary demands of Heritage management, the results are presented in terms of archaeological knowledge, methodological development and management of the archaeological Heritage. Thus, with the outcomes of a concrete practical case we seek to contribute meaningfully to the current debate between the research dimension and the management direction of the Archaeological discipline.

Palabras-clave: Gestión del Patrimonio Cultural. Metodología. Prospección Arqueológica. Arqueología del Paisaje. Evaluación de Impacto Arqueológico. Medidas Correctoras.

Key words: Management of Cultural Heritage. Methodology. Archaeological Survey. Landscape Archaeology. Evaluation of Archaeological Impact. Mitigation Strategy.

\section{INTRODUCCIÓN}

En octubre de 1991 la Dirección Xeral do Patrimonio Histórico e Documental de la Consellería de Cultura de la Xunta de Galicia, en cumplimiento de la normativa española y gallega sobre la protección del Patrimonio Cultural y Arqueológico, solicita del Departamento de Historia I de la Universidad de Santiago de Compostela (en adelante USC) su colaboración para que realizase actuaciones técnicas destinadas a mitigar el Impacto Arqueológico de la 


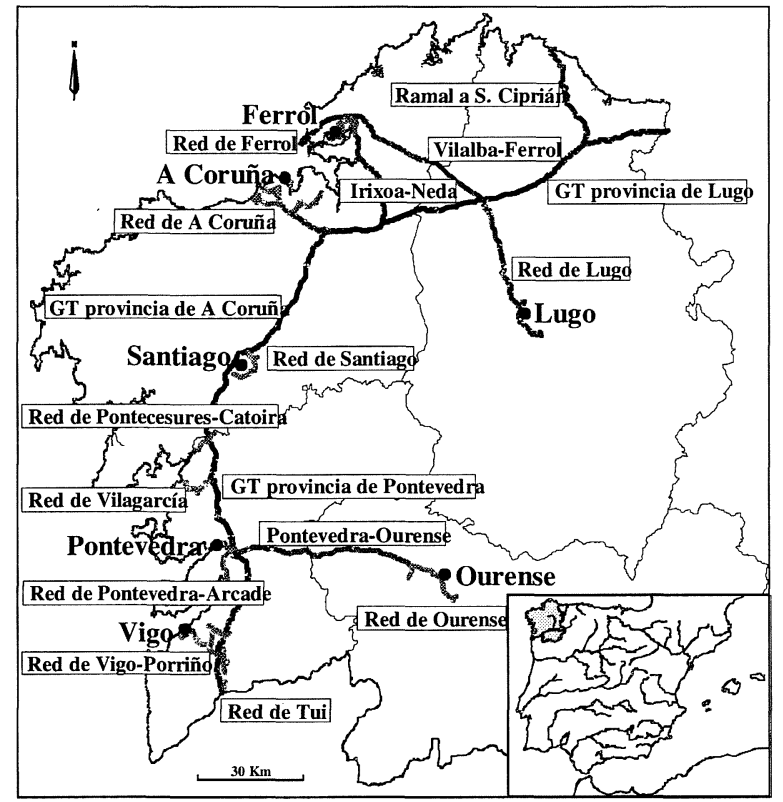

Fig. 1. Localización del área de trabajo. Mapa de la Red de Gasificación de Galicia.

Red de Gasificación de Galicia proyectada por ENAGAS y de las otras dos grandes Obras Públicas previstas los años siguientes en el territorio gallego: el Oleoducto Coruña-Vigo proyectado por CLH y las Autovías Benavente-Lugo-Coruña y Benavente-Ourense-Vigo del MOPT.

El Departamento de Historia I de la USC y concretamente el Prof. Dr. Felipe Criado Boado asumió este encargo y la dirección del proyecto de Evaluación, constituyéndose al efecto el Grupo de Investigación en Arqueoloxía da Paisaxe (en adelante GIArPa). El desarrollo de los trabajos contó desde un principio con el apoyo fundamental del Centro de Transferencia de Tecnología de la USC, marco institucional que facilitó el contacto entre Empresa y Universidad e hizo posible una relación efectiva entre investigación básica y gestión.

Este grupo universitario, partiendo del marco teórico de la Arqueología del Paisaje, diseñó un programa unitario para corregir el impacto arqueológico, rentabilizar en proyectos de investigación la información aportada por las citadas Obras Públicas e integrar las actuaciones arqueológicas de carácter técnico y patrimonial dentro de un proceso de construcción de conocimiento arqueológico.

La finalización en 1997 de los trabajos arqueológicos previos a la construcción del Gasoducto dio paso a la situación actual en la que se presentan como prioritarias tanto la difusión y comunicación de los resultados obtenidos (1) como la investigación del ingente registro arqueológico generado por la Fase de Evaluación de ImpactoArqueológico (2). De este modo, este texto se inscribe en una amplia labor de divulgación (Criado et alii, 1997; Amado et alii, 1998; Ayán y Amado, 1999) con la que se pretende alcanzar un balance crítico de la práctica arqueológica desarrollada en el seno del proyecto objeto de estudio.

A este respecto cabe señalar, en primer lugar, la doble problemática que conlleva un proyecto de estas características. Por un lado, la construcción de la Red de Gasificación de Galicia requería, desde el punto de vista de una Arqueología Preventiva, unos criterios de acción práctica y unas metodologías de intervención para tratar adecuadamente una problemática nueva tanto en Galicia como a nivel del Estado Español (3). Así pues, desde 1991 se daría un notable proceso de avance metodológico cuyo resultado sería el diseño de una práctica de próspección arqueológica superficial adaptada a las características de los proyectos y la formulación de una estrategia global de Evaluación de ImpactoArqueológico con el objetivo de mitigar la afección del trazado sobre el Patrimonio Arqueológico. Teniendo en cuenta esta circunstancia, consideramos relevante reseñar brevemente dichos procedimientos metodológicos.

Por otro lado, el proyecto lleva implícita una compleja problemática como es la necesidad de

(1) Hasta el momento carecíamos de un balance global del conjunto de los estudios de evaluación de impacto de la Red de Gasificación, contándose únicamente con un primer avance provisional de los trabajos llevados a cabo hasta 1993 (González et alii, 1995).

(2) Por Fase de Evaluación de Impacto Arqueológico entendemos el conjunto de trabajos de evaluación relacionados con la Red de Gasificación de Galicia realizados entre 1991 y 1997 . No se trata de un proceso lineal, sino que se pueden identificar tres subfases (1991-1993; 1995 y 1997) en función del desarrollo de los planes de obra. En este sentido cabe señalar que consideramos este período de 1991 a 1997 de una manera convencional para encuadrar el objeto de estudio, pero hay que tener en cuenta que las Evaluaciones de Impacto Arqueológico desarrolladas en 1997 se llevaron a cabo cuando en otros tramos se procedía ya al seguimiento arqueológico de su construcción, por lo que, en este caso, hablamos de fase de Evaluación de Impacto Arqueológico únicamente para facilitar la inteligibilidad del proceso y conferir unidad a la problemática abordada.

(3) Existían contados ejemplos de control arqueológico de redes de gasificación en algunos países de Europa Occidental siendo significativos los casos de los gasoductos construidos a principios de la década de 1980 entre Cork y Dublín (Cleary et alii., 1987) y Tydd St. Giles y Mappower en Wessex-Inglaterra (Catherall et alii., 1984) así como el tramo Génova-Recco realizado entre 1988 y 1989 (Maggi, 1992). El único ejemplo de seguimiento de una obra de trazado lineal en el ámbito español es el programa de excavaciones llevado a cabo en el trazado de la autopista A-19, variante de Mataró (Codex, 1992). 

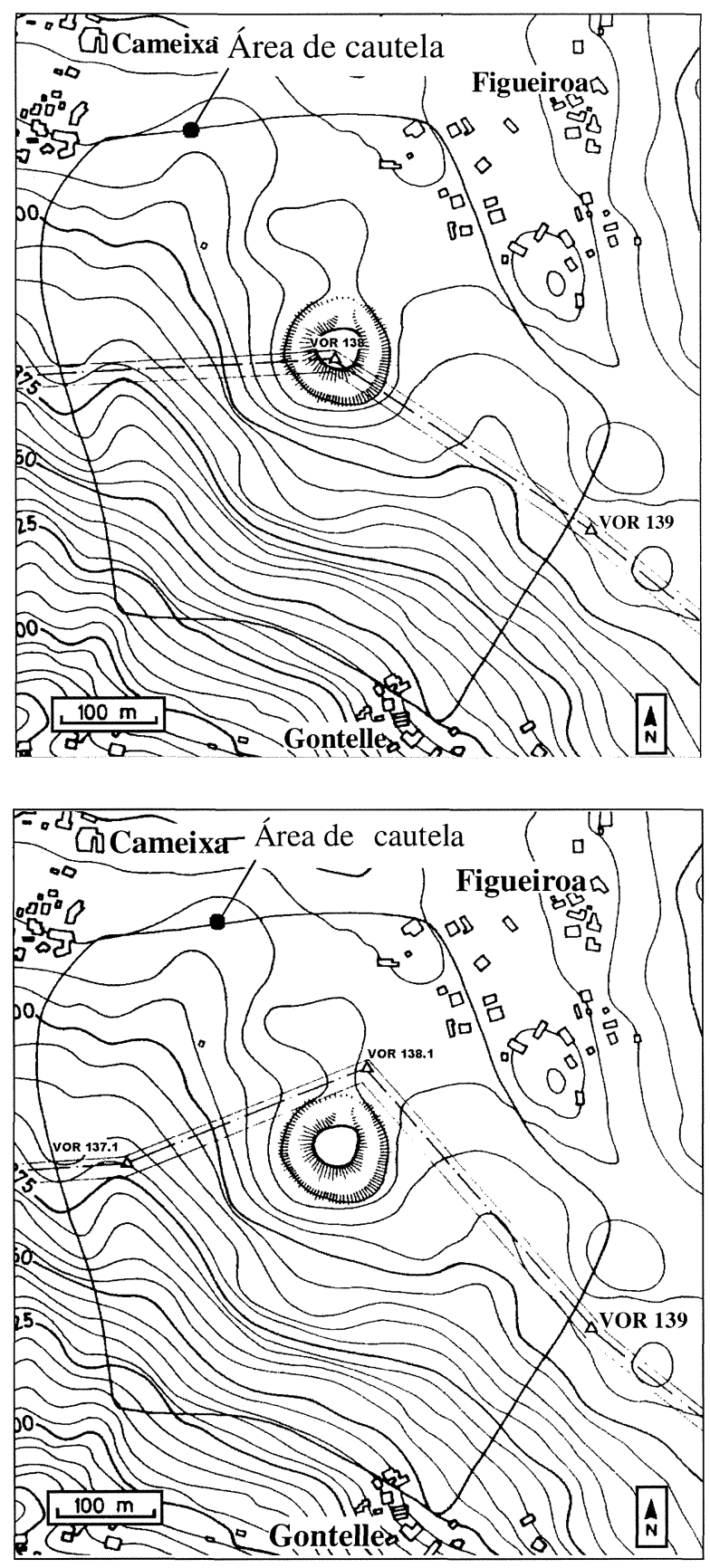

Fig. 2. Un ejemplo de modificación de trazado en fase de Evaluación de Impacto Arqueológico: impacto crítico sobre el castro de Cameixa (Boborás, Ourense).

compatibilizar una intervención sobre el Patrimonio (objetivo práctico) con la generación de conocimiento arqueológico nuevo (objetivos cognoscitivos y teóricos). En este sentido, ofrecemos los resultados arqueológicos, metodológicos y patri- moniales de la Fase de Evaluación de Impacto Arqueológico de la Red de Gasificación de Galicia, con la intención de valorar su verdadero alcance y significación.

\section{DISEÑO Y APLICACIÓN DE UNA METODOLOGÍA DE EVALUACIÓN DE IMPACTO EN LA RED DE GASIFICACIÓN DE GALICIA: 1991-1997}

Desde un principio, la inspección de campo o prospección arqueológica superficial previa al inicio de las obras, llevada a cabo durante la fase de diseño del proyecto definitivo, se presentaba como la estrategia más adecuada para garantizar, en la medida de lo posible, una valoración correcta de las incidencias arqueológicas ocasionadas por los trazados estudiados.

Por lo tanto, dicha prospección fue el presupuesto básico de la estrategia de Evaluación y Corrección de Impacto Arqueológico del Gasoducto; no obstante, a pesar de la claridad de estos postulados iniciales, se mantenía la problemática referida a su alcance y naturaleza, cuestión finalmente solventada con la articulación de un programa de actuación (4) que contemplaba, en un primer momento, dos tipos de prácticas prospectivas adaptadas a los condicionantes y limitaciones impuestos por el registro arqueológico gallego (5):

- Prospección superficial extensiva: catalogación arqueológica a una escala amplia y centrada en la localización de los yacimientos de naturaleza visible o monumental, fundamentalmente, para el caso gallego, túmulos neolíticos, castros de la Edad del Hierro, fortificaciones medievales y sistemas de cultivo pretéritos. Este tipo de trabajo, aunque descubre una mínima parte de los yacimientos afectados, permite, por un lado, evaluar las incidencias arqueológicas de mayor porte y complejidad dentro del marco de la obra proyectada, y por otro, plantear cambios de trazado que, aun siendo de pequeña entidad, contribuyen a solventar problemas arqueológicos importantes.

(4) Los métodos de prospección intensiva aplicados fueron ensayados anteriormente en el proyecto arqueológico de estudio de los paisajes prehistóricos de la sierra de O Bocelo y valle del río Furelos (Melide, Toques, A Coruña) (Criado et alii, 1988; Criado, 1989; Criado et alii, 1992).

(5) Nos referimos al elevado índice de dispersión y la escasa entidad de los núcleos de poblamiento ya en la Prehistoria gallega, así como a la invisibilidad en superficie de la mayoría de los yacimientos arqueológicos (entre el 65 y el $75 \%$ aproximadamente) 


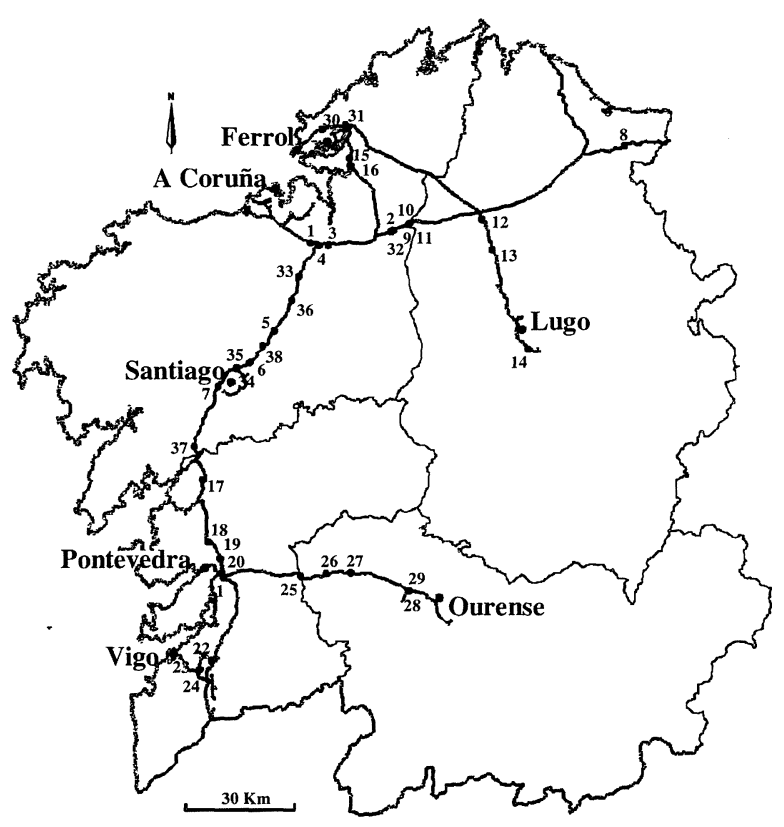

Fig. 3. Yacimientos arqueológicos preservados en fase de Evaluación de ImpactoArqueológico mediante desvíos de trazado:

Desvío de trazado y cambio de orientación de la pista de trabajo: 1: Castro de Mabegondo, 2: Túmulo so Pena Moura, 3: Castro de Callobre, 4: Mámoa da Granxa, 5: Castro de Bouza Longa, 6: Mámoa do Monte da Costa Negra, 7: Castro de Villestro.

Desvío de trazado: 8: Marco da Pena Verde, 9: Necrópole do Galiñeiro, 12: Mámoa de Curros, 13: Mámoa deAs Lagas, 14: Mámoa de Trigás, 15: Castro de Magalofes, 16: Monte do Castro, 17: Petroglifo de Cortiñas, 18: Mámoa Perdida, 19: Mámoa de Monte Barburiña, 20: Mámoas de Monterilla, 21: Mámoas de Monterilla, 22: Castro de Torroso, 23: Mámoas do Mercantil, 24: Mámoas do Mercantil, 25: Mámoa da Moradiña, 26: Mámoa de Torre Mourelle, 27: Castro de Cameixa, 28: Mámoa de As Chairas, 29: Mámoa de As Chairas. Cambio de orientación de la pista de trabajo: 30 : Castro de Vilela, 31: Os Castros, 32: Mámoa de Ferreirós, 33: Castro de Ferrás, 34: Mámoas do Son, 35: Mámoas do Son, 36: Os Castros, 37: Castelo de Monte Villar, 38: Castro de Vilacide.

- Prospección intensiva: catalogación arqueológica a una escala de detalle, local, y centrada en el descubrimiento de restos arqueológicos de todos los períodos. Esta estrategia permite localizar un número importante de los yacimientos existentes y realizar estimaciones fiables sobre las zonas en las que potencialmente pueden localizarse otros no visibles. De este modo se completan los datos ofrecidos por la actuación extensiva anterior y, por otro lado, se contrastan en la práctica unos modelos predictivos para su localización que permiten evaluar de manera preliminar los imprevistos de naturaleza arqueológica surgidos durante la acción de las máquinas.

Estas dos variantes evidenciarían en los primeros estudios de Evaluación de Impacto Arqueológi- co de 1991 y 1992 la necesidad de considerar una metodología de prospección que se amoldase a las características de una obra de trazado lineal como el Gasoducto. Este sería el punto de partida para la formulación de un nueva modalidad dentro de la práctica prospectiva, definida como Prospección Intensiva Selectiva y que se convertiría en el instrumento básico y fundamental de los trabajos arqueológicos de carácter preventivo desarrollados por el GIArPa hasta 1997.

El sistema así denominado consistía fundamentalmente (Méndez et alii, 1995: 294) en una prospección de carácter extensivo de la totalidad del terreno, completada con la selección de determinadas zonas del trazado donde la prospección sería intensiva. Con esta estrategia se esperaba alcanzar un triple objetivo: a) identificar la mayor parte del registro arqueológico visible; b) localizar una muestra significativa de yacimientos invisibles, para así poder contar con una información lo más exhaustiva posible, y c) agilizar el trabajo arqueológico y minimizar el coste del mismo.

El carácter preliminar de estas tareas de prospección constituye, a su vez, un factor prioritario en esta Fase de Evaluación de ImpactoArqueológico, ya que la introducción de las variaciones necesarias en un trazado con anterioridad a la elaboración del proyecto definitivo permite una mejor y más completa corrección de su afección sobre el Patrimonio Arqueológico. Este hecho lo ejemplifica perfectamente la comparación entre la prospección llevada a cabo en la Red de Gasificación con la del Oleoducto. En la Evaluación de Impacto Arqueológico de éste último (Méndez et alii, 1995) la prospección se realizó cuando el proyecto de detalle ya estaba finalizado, lo que hizo especialmente compleja la introducción de modificaciones en el proyecto de trazado. Por el contrario, en el caso del Gasoducto, al encontrarse los tramos en fase de elaboración del proyecto de detalle se pudieron introducir variaciones en el trazado con mucha facilidad, evitándose así la destrucción de yacimientos arqueológicos de gran porte.

Este impacto crítico no sería subsanable de no ser por la flexibilidad que ofrece esta dinámica de trabajo, definida por la colaboración mantenida entre los equipos de GIArPa y las Empresas de Ingeniería que diseñaron los proyectos de trazado. Los trabajos de campo se iniciaban cuando la Empresa contratante aportaba un proyecto de trazado sobre una cartografía de detalle escala 1:5000 y, generalmente, después de que ese trazado hubiese

T. P., 58, n. $^{\circ} 1,2001$ 
sido marcado en el campo. Todo ello facilitaba a los equipos prospectar la traza proyectada, así como la identificación, inventariado y delimitación de los bienes arqueológicos situados en su ámbito de afección.

Cuando se identificaron impactos de carácter crítico, se propuso la modificación del trazado. Las alternativas eran estudiadas por parte de la ingeniería y consensuadas con el Equipo Arqueológico valorando la efectividad de la nueva propuesta. En algunas ocasiones fueron necesarias reuniones específicas para estudiar y adoptar una solución que, en última instancia, debió siempre ser sancionada por la Dirección Xeral do Patrimonio Cultural.

Esta dinámica de colaboración, impuesta por el propio planteamiento y desarrollo de las obras de Gasificación, requería estructurar el trabajo arqueológico de acuerdo con una secuencia operativa a la que se debían amoldar tanto las actuaciones arqueológicas como los informes generados por las mismas. En un primer momento (1991) la Evaluación de Impacto Arqueológico contemplaba cinco fases sucesivas en las que se combinaba tanto el trabajo de gabinete como el de campo:

1. Elaboración y redacción del proyecto arqueológico, así como planteamiento del trabajo de campo a partir del análisis de toda la documentación disponible sobre la zona afectada por el trazado: información arqueológica, cartografía de detalle facilitada por la Empresa, fotografía área y datos toponímicos. Este trabajo permitía elaborar una hipótesis de partida para la localización de yacimientos arqueológicos.

2. Con toda esta información se procedía a una primera inspección del terreno con un doble objetivo: por un lado, estudiar en profundidad el marco geográfico por el que discurre la conducción del gas, y, por otro lado, localizar los yacimientos de mayor envergadura previsiblemente afectados por el trazado.

3. Las incidencias arqueológicas registradas se recogían en unAvance de Informe, que se remitía a la Empresa de Ingeniería encargada del diseño del proyecto de detalle. De este modo, antes de tramitar la expropiación temporal de los terrenos, se podrían realizar los cambios necesarios en el trazado.

4. Una vez definido el primer proyecto de detalle se lleva a cabo una prospección extensiva de la totalidad del trazado y una de carácter intensivo en aquellas zonas donde, de acuerdo con los modelos predictivos e hipótesis propuestas, era probable la aparición de restos de naturaleza arqueológica.
5. La última fase de los trabajos se corresponde con la elaboración del Informe Final en el que se presentan los resultados del estudio de Evaluación de Impacto, siendo integrado en el Proyecto de construcción de la Obra.

Este modelo organizativo contribuyó de manera significativa a la configuración de un sistema de trabajo específico centrado en la problemática arqueológica de la Red de Gasificación. Sin embargo, la inexistencia de criterios de evaluación previos, la ausencia de conceptos claros de impacto, cautela arqueológica y medidas correctoras, así como una muy vaga contextualización de los resultados, limitaban la eficiencia de este tipo de estudios de impacto. Pero la principal dificultad para el desarrollo de esta práctica arqueológica y patrimonial vendría dada por la concepción que se tenía del alcance, naturaleza y finalidad de un estudio de impacto que se reducía a identificar restos, correlacionarlos con las obras, identificar destrucciones y proponer medidas correctoras, pero sin aplicar criterios objetivos previamente explicitados.

El informe final privilegiaba soluciones propias de la Arqueología tradicional como la excavación o el sondeo, sin desarrollar medidas concretas en función de la problemática de la obra más allá del obvio desvío de trazado, constatándose un desajuste evidente con las soluciones prácticas demandadas por la empresa contratante a la hora de establecer las cautelas oportunas durante la fase de obra.

La incidencia negativa de todas estas circunstancias incentivaría la reformulación de la metodología de actuación aplicada hasta el momento. De este modo, en 1992 se establece como principio básico en la Evaluación de ImpactoArqueológico la distancia de las obras con respecto al yacimiento considerado, en función de la normativa legal de reciente aprobación (6) que estipula un radio de protección de $200 \mathrm{~m}$ dentro del cual toda obra debe estar sujeta a un control arqueológico. La adopción de este presupuesto de naturaleza legal en el programa de Evaluación de ImpactoArqueológico ampliaría sus posibilidades de aplicación práctica, ajustándolo a las necesidades del trabajo arqueológico constituido por la prospección superficial del trazado. Desde entonces la estrategia de prospección tipificó y clasificó una serie de zonas de actuación:

(6) Artículo 30 de las Normas Subsidiarias y Complementarias del Planteamiento Urbanístico de las Cuatro Provincias Gallegas. Resolución de la Consellería de Obras Públicas de 14 de mayo de 1991 publicada en el Diario Oficial de Galicia, n 116 y ss., de fecha 19 de junio de 1991. 
1. Zona o Banda de afección: sobre la que la obra actuará de forma directa (terrenos a desmantelar, tráfico de maquinaria, obras auxiliares, zonas de expropiación permanente) más los terrenos de su entorno en un radio de $50 \mathrm{~m}$, diferenciándose a su vez otras dos bandas: a) una de 0 a $18 \mathrm{~m}$ a cada lado del trazado, englobando la superficie afectada de forma directa por la construcción. La práctica prospectiva planteada es fundamentalmente de carácter intensivo; b) banda que abarca desde los $18 \mathrm{~m}$ a los $50 \mathrm{~m}$ de distancia al trazado donde el trabajo arqueológico combina una prospección extensiva de toda la superficie y otra intensiva en zonas seleccionadas en función de las pautas y regularidades marcadas por el registro arqueológico gallego.

2. Zona o Banda de incidencia: área indirectamente afectada por el acceso de maquinaria, entre los 50 y los 200 m medidos a partir del límite exterior de la pista de obra; aquí generalmente la inspección es extensiva.

3. Zona o Banda de muestreo: a partir de los $200 \mathrm{~m}$. Su consideración deriva de cuatro razones fundamentales: una legal, ya citada; otra metodológica, como es completar la evaluación del Patrimonio Arqueológico afectado por las dos bandas anteriores. Otros motivos se relacionan directamente con la propia línea de investigación seguida, como la necesidad prioritaria de integrar los yacimientos documentados en la prospección dentro de su marco arqueogeográfico. Finalmente el objetivo es disponer de información del entorno para estudiar las posibles opciones en el caso de que fuese necesario variar el trazado.

Esta filosofía de actuación permitió desarrollar un sistema organizativo válido para adecuar las distintas variables que confluyen determinantemente en un trabajo de Evaluación de Impacto Arqueológico de esta naturaleza: a) alcance, grado e intensidad de los trabajos de prospección superficial; b) características técnicas de la Obra; c) impacto arqueológico de la misma y d) comunicación y presentación de resultados.

La principal aportación de esta reformulación metodológica fue una mejor contextualización del registro arqueológico documentado, lo que redujo parcialmente la incorrección anterior debida al tratamiento de unos datos en buena medida sesgados por la restricción del marco de actuación a las proximidades del trazado. Sin embargo, aún así, se mantendrían algunas de las carencias más significativas del programa de trabajo inicialmente diseñado, tales como la falta de una tabla tipológica de impac- tos o la necesidad urgente de desarrollar una estrategia de corrección más flexible y acorde con las exigencias de la obra.

Esta doble problemática actuó como un incentivo crucial para un nuevo replanteamiento metodológico, materializado en los estudios de impacto realizados entre 1995 y 1997 , que se puede considerar como el avance más significativo dentro del proceso de desarrollo y definición de una metodología específica de Evaluación de Impacto Arqueológico. Esta reorientación consistió, fundamentalmente, en una aproximación de carácter interdisciplinar hacia un ámbito de estudio, en principio, aparentemente alejado de la Arqueología tradicional, como son los modelos metodológicos de Evaluación de ImpactoAmbiental propuestos en los últimos años (Suárez Cardona, 1989; Gómez Orea, 1994; Conesa, 1995) a raíz del establecimiento de una legislación reguladora (7) sobre la materia a mediados de los 80 .

La marcada similitud existente entre los campos de actuación y estudio de la Evaluación de Impacto Ambiental y de la Evaluación de Impacto Arqueológico (8) haría especialmente factible un productivo acercamiento interdisciplinar. De este modo, el incipiente desarrollo en la década de 1980 de los estudios de Evaluación de ImpactoAmbiental, les situaba como modelo a seguir por una práctica arqueológica preventiva todavía en ciernes, y carente de una metodología específica para abordar las nuevas problemáticas planteadas.

La estrategia de Evaluación y Corrección de Impacto desarrollada por el GIArPa desde 1995 se benefició directamente de estas aportaciones a la hora de configurar un modelo de evaluación específico aplicable a la problemática arqueológica. Sin embargo, es importante destacar que este acercamiento no consistió en una simple transposición de presupuestos de una disciplina a otra, sino que todo ello conllevó un notable esfuerzo metodológico de adaptación a las necesidades de la Evaluación de Impacto Arqueológico. En este sentido fueron cua-

(7) Real Decreto Legislativo 1302/86, de 28 de junio, y en Real Decreto $1131 / 88$, de 30 de septiembre, por el que se aprueba el Reglamento para la ejecución del Decreto citado.

(8) Su objetivo es responder adecuadamente a las demandas impuestas por un contexto común en el que convergen una mayor concienciación social en materia de protección y conservación del Medio Ambiente y del Patrimonio Cultural, la promulgación de una normativa legal reguladora de las actuaciones de carácter preventivo (ver nota 7), o el fomento institucional de una política medioambiental y patrimonial no sólo centrada en la protección sino también en la revalorización y rentabilización de esos bienes. 
tro los aspectos integrados en el programa de evaluación propuesto por el GIArPa:

Conceptualización: las dos nociones básicas de la Evaluación de ImpactoArqueológico adoptan el marco propuesto por los modelos de Evaluación de Impacto Ambiental:

- Impacto arqueológico: definido como toda alteración o agresión concreta que un proyecto presenta sobre los elementos que conforman el PatrimonioArqueológico como componente específico del MedioAmbiente.

- Evaluación de impacto arqueológico: proceso de análisis por el que se identifica (relaciones causa-efecto), predice (cuantifica), valora (interpreta), previene (corrige de forma preventiva) y comunica (participación pública) el impacto arqueológico de un proyecto en el caso de que se ejecute.

Secuencia operativa: la consideración del trabajo de Evaluación de ImpactoArqueológico como un proceso constituido por diversas fases sucesivas de actuación (análisis de proyecto, valoración de inventario, evaluación de impacto y propuesta de medidas correctoras) se inspira directamente en los estudios de Evaluación de Impacto Ambiental.

Tipología de impactos: Esta aportación fundamental del modelo de Evaluación de ImpactoAmbiental permitió solventar definitivamente una de las principales carencias de las que adolecían los estudios de Evaluación de Impacto Arqueológico del Gasoducto desarrollados entre 1991-1995. Se adoptó la clasificación y denominaciones de impactos recogidas en la normativa legal (9) y estudios de gestión ambiental, aunque la asignación de cada uno de los tipos de impacto a los yacimientos documentados se haría en función de su distancia al trazado y su situación dentro de las bandas de trabajo:

a) Impacto crítico: aplicado a los elementos arqueológicos situados en la franja de explanación o remoción de tierras, hasta los $20 \mathrm{~m}$ de distancia. Estos elementos serán destruidos o gravemente alterados por las obras.

b) Impacto severo: para los elementos situados entre los 20 y $50 \mathrm{~m}$, donde se pueden producir incidencias como resultado de la actuación de los diversos agentes y acciones de la obra (paso de maquinaria, construcciones auxiliares, etc.).

(9) Se emplearía por primera vez en los trabajos de EIArq de las autovías del MOPT (Méndez et alii, 1995: 30) y en el estudio de EIArq de la Red de distribución de Ourense realizado a finales de 1993, respectivamente. c) Impacto moderado: para los elementos situados entre los $50 \mathrm{y}$ los $150 \mathrm{~m}$, previsiblemente dañados por imprevistos técnicos o alteraciones accidentales.

d) Impacto compatible: para los restos localizados entre los 150 y $200 \mathrm{~m}$, que pueden verse accidentalmente dañados y en todo caso afectado su entorno.

e) Finalmente se considera la opción no afecta para aquellos elementos localizados fuera de la banda de $200 \mathrm{~m}$ durante el transcurso de los trabajos e incluidos dentro del inventario.

Noción de medidas correctoras: el desarrollo de un programa de vigilancia ambiental basado en la articulación de todo un conjunto de medidas de corrección claramente definidas serviría de punto de partida para la formulación de una estrategia de corrección de impacto arqueológico que superase la imprecisión y escaso alcance de las propuestas de actuación consideradas en los primeros estudios de Evaluación dé Impacto Arqueológico. En este sentido se definieron una serie de medidas correctoras diseñadas con el fin de mitigar y minimizar en la medida de lo posible el impacto previsto en fase de evaluación. Aunque estas medidas se aplican de forma flexible a cada caso concreto en función de la evaluación de cada elemento, se diferenciarían fundamentalmente dos modalidades o tipos:

a) Cautelas efectivas: referidas a yacimientos ya localizados o al entorno de los mismos, implican una serie de medidas de protección generalmente establecidas por vía administrativa, mediante las cuales se prohibe el movimiento de tierras y/o el tránsito de maquinaria hasta que se ejecuten las medidas correctoras o compensatorias para paliar el impacto.

b) Cautelas preventivas: dirigidas principalmente a los arqueólogos encargados del seguimiento de las obras. Este tipo de propuestas toman forma ante impactos hipotéticos, definidos con base en los estudios espaciales y patrones de emplazamiento, delimitando áreas de riesgo en aquellas zonas en las que es altamente probable la localización de yacimientos o restos arqueológicos.

La introducción de todos estos criterios permitiría la puesta a punto de una estrategia de Evaluación de Impacto con una metodología bien definida que, si bien constituye el punto de llegada provisional de todo el proceso de desarrollo experimentado durante la Fase de Evaluación de Impacto Arqueológico, fue el resultado provisional de una línea de investigación promovida por el GIArPa que sigue profundi- 
zando actualmente en la sistematización de herramientas y estrategias de trabajo que faciliten la resolución, desde la disciplina arqueológica, de la problemática patrimonial presentada por los proyectos de construcción de Obras Públicas.

Esta metodología de evaluación, expuesta en trabajos recientes (Martínez et alii, 1997: 41-46; Criado et alii, 1998;Amado et alii, 1998: 153-164), sería llevada a la práctica, por primera vez, en los estudios de Evaluación de ImpactoArqueológico de Parques Eólicos en Galicia por un equipo de GIArPa desde mayo de 1995 (Villoch y Barreiro, 1997; Barreiro y Villoch, 1997). A pesar de que la problemática planteada difiere en enorme medida de la del Gasoducto, la experiencia aportada por estos estudios permitió comprobar su efectividad y resultados, marcando las directrices básicas a seguir en la Evaluación de ImpactoArqueológico de los tramos de la Red de Gasificación prospectados en 1997. En estos últimos trabajos, la Evaluación de Impacto Arqueológico aparece definida como una práctica arqueológica específica inserta en un programa de gestión integral del Patrimonio articulado a la manera de una cadena valorativa. Este programa parte de una propuesta teórica (Criado 1996a, b, c) que aboga por una reconversión de la Arqueología en tecnología, en disciplina de gestión del Patrimonio Arqueológico y práctica valorativa, como medio para unificar la práctica arqueológica y superar la contraposición entre gestión e investigación vigente en la actualidad. En este contexto la evaluación ya no se reduce a una intervención de carácter técnico sobre el Patrimonio, sino que amplía su función y sentido práctico, siendo concebida como un instrumento básico para llevar a cabo la valoración arqueológica y patrimonial de los bienes culturales afectados.

\section{LA EVALUACIÓN DE IMPACTO COMO PRÁCTICA PREVENTIVA RENTABLE PARA LA PROTECCIÓN DEL PATRIMONIO ARQUEOLÓGICO}

Los trabajos previos, basados en prospecciones superficiales de los trazados proyectados, resultaron especialmente efectivos a la hora de registrar, evaluar y mitigar su impacto arqueológico. Se identificaron un total de 159 impactos, lo que significa que, sin el control arqueológico de las obras proyectadas, el $62 \%$ de los yacimientos documentados habrían sufrido un impacto de carácter irreversible.

\begin{tabular}{|l|c|}
\hline \multicolumn{1}{|c|}{ TIPO DE IMPACTO } & $\mathbf{N}^{\circ}$ DE IMPACTOS \\
\hline Crítico & 40 \\
\hline Severo & 39 \\
\hline Moderado & 22 \\
\hline Compatible & 58 \\
\hline
\end{tabular}

Tab. 1. Impactos registrados en fase de Evaluación de Impacto Arqueológico.

Asimismo cabe destacar que una obra de estas características, debido a la notable dispersión del poblamiento gallego ya desde la Prehistoria, ocasionaría efectos muy considerables sobre el Patrimonio de no haber mediado el correspondiente programa de Evaluación y Corrección de Impacto.

Dicho programa contempló, por un lado, la adopción de ocho tipos de medidas correctoras aplicadas en función de la especificidad de cada uno de los 159 casos concretos y, por otro lado, el establecimiento de 74 áreas de cautela arqueológica a considerar durante la fase de construcción de la obra.

\begin{tabular}{|l|c|}
\hline \multicolumn{1}{|c|}{ MEDIDAS CORRECTORAS } & N $^{\circ}$ DE YACIMIENTOS \\
\hline Desvíos de trazado & 29 \\
\hline Cambio de orientación de la pista de obra & 16 \\
\hline Prospección Intensiva/Selectiva & 27 \\
\hline Prospección Físico-Química & 6 \\
\hline Excavación & 6 \\
\hline Sondeos & 8 \\
\hline Señalización & 86 \\
\hline Control/Seguimiento & 116 \\
\hline
\end{tabular}

Tab. 2. Medidas correctoras adoptadas en fase de Evaluación de Impacto Arqueológico.

En este sentido, el logro más rentable (tanto desde el punto de vista económico como patrimonial) de los trabajos de evaluación previos a la construcción de la obra fue evitar la destrucción total o parcial de 38 yacimientos arqueológicos de naturaleza visible y monumental mediante la modificación de trazado y/o el cambio de sentido de la pista de obra. Estos yacimientos eran sobre todo castros y túmulos megalíticos, lo que supone que las afecciones más graves sobre el Patrimonio Arqueológico fueron evitadas durante la fase de diseño de los trazados.

Además los trabajos de Evaluación de Impacto Arqueológico permitieron subsanar en mayor o menor grado otros 50 impactos directos (11 críticos y 39 severos). El gran número de incidencias arqueológicas solventadas y los yacimientos arqueológicos preservados avalan la efectividad y necesi- 


\begin{tabular}{|c|c|c|c|c|c|c|c|c|c|}
\hline \multirow{2}{*}{ TRAMOS } & \multirow{2}{*}{ CóDIGo } & \multirow{2}{*}{ INGENIERÍA } & \multirow{2}{*}{ LONGITUD } & \multicolumn{4}{|c|}{ YACIMIENTOS POR DISTANCIAS } & \multirow{2}{*}{$\begin{array}{c}\text { DESVÍos } \\
\text { DE } \\
\text { TRAZADO }\end{array}$} & \multirow{2}{*}{$\begin{array}{c}\text { MEDIDAS } \\
\text { CORRECTORAs }\end{array}$} \\
\hline & & & & TOTAL & $0-20 \mathrm{~m}$ & $20-50 \mathrm{~m}$ & $50-200 \mathrm{~m}$ & & \\
\hline $\begin{array}{l}\text { Gasoducto de Transporte de la } \\
\text { provincia de Lugo }\end{array}$ & $92 \mathrm{G} 01$ & INTECSA & $82 \mathrm{~km}$ & 31 & 7 & 3 & 9 & 3 & 44 \\
\hline $\begin{array}{l}\text { Gasoducto de transporte de la } \\
\text { provincia de A Coruna }\end{array}$ & $92 \mathrm{G} 04$ & INITEC & $103 \mathrm{~km}$ & 29 & 7 & 12 & 10 & 14 & 38 \\
\hline $\begin{array}{l}\text { Gasoducto de transporte de la } \\
\text { provincia de Pontevedra }\end{array}$ & $92 \mathrm{G} 05$ & INITEC & $85 \mathrm{~km}$ & 26 & 6 & 7 & 14 & 6 & 34 \\
\hline Ramal de S. Cibrán & $92 \mathrm{G} 02$ & INTECSA & $31 \mathrm{~km}$ & 2 & 0 & 1 & 1 & 0 & 3 \\
\hline Ramal Vilalba-Ferrol & $92 \mathrm{G} 10$ & INITEC & $64 \mathrm{~km}$ & 24 & 3 & 3 & 18 & 2 & 15 \\
\hline $\begin{array}{c}\text { Ramal Vilalba-Lugo } \\
\text { y Red de Lugo }\end{array}$ & $92 \mathrm{G} 09$ & DOM & $49 \mathrm{~km}$ & 18 & 2 & 5 & 11 & 3 & 17 \\
\hline Ramal Irixoa-Neda & $\begin{array}{l}95 \mathrm{G} 05 \\
95 \mathrm{G} 18 \\
\end{array}$ & HEYMO & $29 \mathrm{~km}$ & 3 & 1 & 1 & 1 & 2 & 5 \\
\hline Ramal Pontevedra-Ourense & $93 \mathrm{G} 03$ & SERELAND & $56 \mathrm{~km}$ & 35 & 4 & 11 & 20 & 3 & 29 \\
\hline Red de Ferrol & $92 \mathrm{G} 08$ & INITEC & $16 \mathrm{~km}$ & 0 & 0 & 0 & 0 & 0 & 0 \\
\hline Red de A Coruna & $92 \mathrm{G} 06$ & INITEC & $64 \mathrm{~km}$ & 29 & 7 & 12 & 10 & 1 & 8 \\
\hline Red de Santiago & $92 \mathrm{G} 07$ & INITEC & $16 \mathrm{~km}$ & 6 & 0 & 0 & 6 & 0 & 5 \\
\hline Red de Pontecesures-Catoira & $97 \mathrm{G} 15$ & SERENSA & $9 \mathrm{~km}$ & 7 & 3 & 2 & 2 & 0 & 3 \\
\hline Red de Vilagarcía & $97 \mathrm{G} 14$ & HEYMO & $15 \mathrm{~km}$ & 7 & 3 & 2 & 2 & 0 & 11 \\
\hline Red de Pontevedra-Arcade & $97 \mathrm{G} 13$ & SERENSA & $16 \mathrm{~km}$ & 2 & 0 & 1 & 1 & 0 & 2 \\
\hline Red de Vigo-Porrino & $97 \mathrm{G} 12$ & SERENSA & $43 \mathrm{~km}$ & 45 & 15 & 11 . & 19 & 2 & 53 \\
\hline Red de Tui & $97 \mathrm{G} 16$ & SERENSA & $3 \mathrm{~km}$ & 4 & 1 & 0 & 3 & 0 & 6 \\
\hline Red de Ourense & $93 G 12$ & IDOM & $23 \mathrm{~km}$ & 10 & 4 & 1 & 5 & 2 & 14 \\
\hline
\end{tabular}

Tab. 3. Síntesis de la Evaluación de ImpactoArqueológico de la Red de Gasificación de Galicia. Se indica el tramo estudiado, el código de proyecto, la empresa de ingeniería contratante, la longitud del tramo, el número de yacimientos registrados en cada una de las bandas de prospección, el número de desvíos de trazado y medidas correctoras adoptadas en cada tramo.

dad de ejecución de la Evaluación de Impacto Arqueológico en obra de construcción.

\section{LA EVALUACIÓN DE IMPACTO COMO PRÁCTICA GENERADORA DE CONOCIMIENTO ARQUEOLÓGICO}

La prospección superficial supuso no sólo el descubrimiento de nuevos yacimientos sino también la contextualización espacial y estudio de aquellos ya catalogados con anterioridad situados en el área de afección del trazado. En el momento del diseño y proyección de la red de gasoductos durante la fase de Evaluación de Impacto Arqueológico se han documentado 208 yacimientos arqueológicos, 26 estructuras de carácter etnográfico y 20 elementos del Patrimonio Histórico-Artístico, en su mayor parte de época medieval. Del total de 254 elementos considerados en el inventario, 159 se verían afectados por las obras. De estos 254 elementos patrimoniales, 233 se ubican dentro de las franjas prospectadas, 113 en la banda de afección (entre los $0-50 \mathrm{~m})$ y 120 en la banda de incidencia $(50-200 \mathrm{~m})$.
El registro arqueológico generado, con el descubrimiento de puntos y yacimientos de carácter inédito, nos da una primera idea de la importancia y del alcance de estos trabajos de evaluación. Así mismo, su documentación en la banda de 200 m permitiría contextualizar la información generada $a$ posteriori durante la fase de seguimiento y control a pie de obra.

\begin{tabular}{|l|c|}
\hline TIPOLOGÍA Y ADSCRIPCIÓN CULTURAL & $\mathbf{N}^{\circ}$ DE YACIMIENTOS \\
\hline Yacimientos paleolíticos/epipaleolíticos & 5 \\
\hline $\begin{array}{l}\text { Asentamientos al aire libre } \\
\text { neolíticos/calcolíticos }\end{array}$ & 4 \\
\hline Túmulos megalíticos & 146 \\
\hline Asentamientos de la Edad del Bronce & 1 \\
\hline Petroglifos de la Edad del Bronce & 8 \\
\hline Castros de la Edad del Hierro & 35 \\
\hline Yacimientos romanos & 4 \\
\hline Elementos del Patrimonio Historico-Artístico & 20 \\
\hline Elementos Etnograficos & 26 \\
\hline Indeterminados & 4 \\
\hline Otros & 1 \\
\hline
\end{tabular}

Tab. 4. Inventario de yacimientos documentados en fase de Evaluación de Impacto Arqueológico. 
No obstante, el valor arqueológico y de investigación de los estudios de impacto no se reduce al mero ámbito de la catalogación o inventario de los yacimientos arqueológicos ubicados en las áreas atravesadas por el trazado. La verdadera rentabilidad en términos de investigación del control arqueológico del Gasoducto viene dada, fundamentalmente por el hecho de ofrecer una oportunidad única de obtención de información contextualizada sobre zonas, yacimientos y períodos arqueológicos prácticamente desconocidos hasta la actualidad.

En líneas generales, los datos aportados por la prospección establecen las bases adecuadas para el desarrollo de diversos trabajos de investigación centrados en diferentes problemáticas hasta el momento apenas tratadas por la Arqueología gallega (10). El muestreo sistemático y la información registrada bajo un criterio unitario durante los trabajos servirían de punto de partida para estudiar los diferentes patrones de distribución de los asentamientos prehistóricos, las condiciones de emplazamiento así como la evolución del poblamiento a lo largo de la Historia en diversas zonas de Galicia (11).

El descubrimiento de algunos asentamientos al aire libre que abarcan desde el Epipaleolítico al Neolítico Final y la Edad del Bronce, clarifica aspectos inéditos de la Prehistoria Reciente gallega como es el uso del suelo o el modelo de poblamiento vigente en dichos períodos culturales, prácticamente desconocidos en el ámbito de la investigación arqueológica del Noroeste (Lima y Prieto, 1999; Méndez, 1994; 1998).

Por otro lado, el trazado del Gasoducto ha jugado un papel importante en el avance y renovación de la investigación sobre temáticas que gozan de una mayor tradición en la Arqueología gallega, como la distribución de necrópolis megalíticas (Villoch, 1995; Parcero, 1998; Criado, 1999), el emplazamiento y análisis formal de los petroglifos (Santos, 1998; Santos y Criado, 1998) o la cultura material cerámica (12) pre y protohistórica (Cobas et alii, 1998; Cobas y Prieto, 1999).

(10) Algunos de estos resultados están siendo publicados en las series TAPA (Trabajos en Arqueología del Paisaje) y CAPA (Criterios y Convenciones en Arqueología del Paisaje), autoeditadas por nuestro grupo de investigación.

(11) Este estudio, actualmente en curso, desarrolla el modelo establecido por X. Amado en su Tesis de Licenciatura (Análise da distribución diferencial de xacementos arqueoloxicos en Galiza desde unha escala de síntese, leída en la USC en noviembre de 1995) a partir del registro arqueológico generado por la construcción del Oleoducto Coruña-Vigo.

(12) El registro empírico aportado por la Red de Gasificación ha servido de base para la tesis doctoral de la investigadora de
También se abren nuevas expectativas de investigación en la Edad del Hierro. Se ha identificado un número significativo de poblados fortificados que se verían afectados por las obras de manera más o menos directa en su perímetro externo. Esta circunstancia hace viable el registro de valiosa información referida a la probable existencia de estructuras agrícolas, terrazas y campos de cultivo exteriores asociados a los yacimientos castreños (Criado y Parcero, 1996; Parcero, 1997, 1999).

Finalmente la prospección superficial ha permitido establecer las bases para una delimitación y definición de áreas de interés arqueológico, etnográfico e histórico. Los datos recogidos ofrecen enormes posibilidades para iniciar una línea de investigación en Arqueología del paisaje rural tradicional gallego, ya que el Gasoducto atraviesa toda la variedad de paisajes agrarios de Galicia, de ahí que la información recogida en prospección referente a sistemas de cultivo, ocupación del espacio tradicional, red de caminos, parcelación, sistemas hidráulicos, etc... presentes en las zonas de estudio, sirva como fuente primaria inestimable para desarrollar trabajos de investigación en esa línea a una escala macroespacial (13).

En líneas generales, creemos que la Evaluación de Impacto Arqueológico de una Obra Pública de las características de la Red de Gasificación de Galicia, realizada bajo los parámetros de una línea de investigación enArqueología del Paisaje, ha permitido, mediante su incidencia sobre yacimientos, estructuras y puntos arqueológicos, incrementar nuestro conocimiento sobre el registro arqueológico gallego a partir de la información obtenida, y movilizar el peligro de destrucción y afección sobre el $\mathrm{Pa}$ trimonio como fuerza generadora de conocimiento arqueológico sobre temáticas inéditas.

\section{CONSECUENCIAS METODOLÓGICAS Y ORGANIZATIVAS DE LA FASE DE EVALUACIÓN DE IMPACTO}

La configuración de metodologías de evaluación y criterios de corrección del Impacto Arqueológi-

GIArPa Pilar Prieto: Forma, estilo y contexto en la cultura material de la Edad del Bronce gallega: cerámica campaniforme y cerámica no decorada, leída en la USC en abril de 1998.

(13) Esta temática está siendo estudiada desde una perspectiva etnoarqueológica por nuestra compañera Paula Ballesteros. Un primer avance de su investigación aparecerá próximamente publicado en un volumen de la serie TAPA (Contribución a una Arqueología Rural de Galicia). 
co se presenta como la principal aportación del proyecto de la Evaluación de Impacto Arqueológico del Gasoducto gallego. El vacío metodológico existente a nivel del Estado Español en lo tocante a esta problemática, si bien en un principio constituyó un enorme condicionante, a largo plazo actuaría como un incentivo fundamental para el desarrollo de una metodología específica de Evaluación de Impacto Arqueológico.

Aunque este modelo metodológico comenzó a ser formulado durante la construcción del Oleoducto de CLH Coruña-Vigo (Méndez et alii, 1995; Criado et alii, 1995) y el control arqueológico de los primeros tramos de las Autovías (Méndez et alii, 1995), sería a lo largo de los trabajos de Evaluación de ImpactoArqueológico de la Red de Gasificación de Galicia entre 1991 y 1997 donde se constata el proceso de planteamiento y desarrollo de esta nueva metodología, un proceso que se puede definir como el paso de la improvisación, en sentido estricto, a la configuración de un programa de gestión integral del Patrimonio Arqueológico.

Así pues, en un primer momento se aprecia una concepción hasta cierto punto preteórica de la Evaluación de Impacto Arqueológico: los estudios de impacto se reducían a la localización de restos arqueológicos y a la identificación de destrucciones o afecciones directas del trazado sobre dichos elementos, se carecía de criterios objetivos para definir los impactos detectados y proponer medidas de corrección para cada caso concreto, etc...

La realización de los primeros trabajos de evaluación entre 1991 y 1993 evidenció claramente estas carencias, lo que conllevaría el diseño de un sistema de trabajo y una metodología rigurosa de evaluación adaptada a la problemática presentada por una obra de trazado lineal de las características del Gasoducto. Las actuaciones llevadas a cabo en 1995 y 1997 plasmarían ya esta nueva reorientación metodológica en la que la Evaluación de Impacto Arqueológico es concebida como una práctica teórico-práctica especializada dentro del ámbito de la Arqueología aplicada, con unos presupuestos teórico-metodológicos, unos criterios y convenciones propias que permiten intervenir de manera productiva, efectiva y ágil en la evaluación y corrección del impacto arqueológico sobre el Patrimonio Cultural.

Por consiguiente, el esfuerzo metodológico realizado por el GIArPa en los últimos ocho años ha conllevado la génesis y desarrollo de una metodología precisa para trabajar en Evaluación de Impacto Arqueológico como un campo específico dentro de una actividad arqueológica adaptada a las exigencias patrimoniales planteadas por Obras Públicas como el Gasoducto.

En este sentido, la fase de Evaluación de Impacto Arqueológico de la Red de Gasificación de Galicia ha permitido constatar dos hechos importantes desde el punto de vista metodológico:

Por un lado, la experiencia aportada por los trabajos de Evaluación de ImpactoArqueológico pone de manifiesto que la máxima rentabilidad en términos de protección del Patrimonio y prevención de Impacto se alcanza si la Evaluación se incorpora en el proceso de toma de decisiones con anterioridad al inicio de la construcción. Este tipo de actuaciones preliminares permite reducir en diverso grado el impacto arqueológico de una obra, pero no una plena efectividad. Esta limitación viene dada, fundamentalmente, por tratarse de trabajos arqueológicos de carácter superficial, lo que le resta eficacia teniendo en cuenta la invisibilidad de la mayor parte del registro arqueológico.

En definitiva, la experiencia aportada por los trabajos relacionados con la Red de Gasificación demuestra la necesidad de establecer como medida imprescindible de corrección la realización de un Seguimiento Arqueológico directo y continuado del proyecto en la fase de remoción de tierras para atenuar de manera efectiva el impacto real sobre el Patrimonio Arqueológico de este tipo de Obras.

En otro orden de cosas, el proyecto actuó como marco global para la adaptación de la prospección arqueológica superficial a las exigencias y condicionantes impuestos por las grandes Obras Públicas de trazado lineal, dando lugar a una nueva herramienta de trabajo, a una práctica prospectiva específica que cubriese esa carencia dentro del marco de la Arqueología Preventiva: la Prospección Intensiva Selectiva, práctica generadora de conocimiento en sí misma y altamente rentable.

A su vez, los trabajos de prospección han puesto de manifiesto la viabilidad de un programa de intervención sobre el Patrimonio basado en la aplicación de estrategias propias de lo que se ha denominado una Arqueología Blanda (Criado, 1995: 256-257) con el objetivo de generar conocimiento arqueológico en el marco de actuaciones de carácter patrimonial. De este modo, se constata que la rentabilidad y efectividad de este tipo de actuaciones se alcanza partiendo, por un lado, de una asunción de los condicionantes impuestos por la obra en cuestión y, por otro lado, de un esfuerzo de adaptación de la disciplina arqueológica, desarrollando 
instrumentos metodológicos prácticos que respondan a las exigencias planteadas por la problemática abordada.

Otra notable consecuencia práctica de orden metodológico ha sido la aplicación en labores de evaluación de impacto de modelos predictivos de localización de yacimientos (basados en el análisis de la configuración geográfica de la zona afectada, las condiciones de emplazamiento de los yacimientos y la documentación arqueológica existente) diseñados para anteriores proyectos de investigación en Arqueología del Paisaje. A este respecto el desarrollo de los estudios de Evaluación de Impacto Arqueológico conllevaría un perfeccionamiento de estos sistemas predictivos, permitiendo realizar estimaciones viables de forma ágil sobre la existencia de yacimientos arqueológicos en zonas afectadas por el trazado de la red de Gasificación; así mismo, estas previsiones orientarían y simplificarían el desarrollo de las estrategias de prospección, con lo que ello supone para una minimización de los costes implicados en la resolución de imprevistos de naturaleza arqueológica y una maximización del trabajo y del conocimiento generado por una actuación de este tipo y características.

Por otro lado, se ha visto que el futuro de la Arqueología, el éxito de su reconversión y adaptación a las exigencias planteadas por la protección e intervención sobre el Patrimonio Cultural, pasa, en cierta medida, por una necesaria aproximación interdisciplinar que permita la incorporación efectiva de metodologías y recursos tecnológicos desarrollados en otras especialidades. Con esto no queremos decir que la Arqueología haya tenido que renunciar a un desarrollo metodológico autónomo, ni mucho menos, sino que la adopción y adaptación de metodologías de actuación procedentes de otros campos de investigación orientadas a problemas prácticos contribuyen de manera significativa y facilitan dicho desarrollo.

Así pues, el caso concreto analizado en nuestro trabajo, esto es, el acercamiento desde la Arqueología al ámbito de estudio de la GestiónAmbiental y la Evaluación de Impacto Ambiental, creemos que constituye un buen ejemplo de esta necesaria apertura y colaboración de la disciplina arqueológica con el objetivo de cubrir determinados vacíos de orden metodológico presentes en la investigación aplicada hasta hace muy poco, como era la ausencia de una metodología específica de Evaluación de Impacto Arqueológico. En este sentido hemos constatado cómo la incorporación de procedimien- tos metodológicos específicos de la Evaluación de Impacto Ambiental no constituyó un mero trasvase mecánico, sino que marcó la pauta e incentivó todo un esfuerzo de desarrollo metodológico muy beneficioso en aras del avance de la teoría y la práctica arqueológicas.

Finalmente cabe destacar el papel jugado por la legislación vigente en Galicia en materia de protección del Patrimonio Cultural como un incentivo crucial para la formulación de metodologías específicas de Evaluación y Corrección de Impacto Arqueológico. En este sentido la normativa legal establecida no actuó como mero marco condicionante de la práctica arqueológica, sino que contribuyó de manera determinante al avance metodológico experimentado desde 1991 y a la aplicación, en este caso al menos, de una política preventiva eficaz (14). De este modo, aspectos recogidos en dicha normativa como la obligatoriedad de realizar estudios valorativos del riesgo de afección de proyectos de obra sobre yacimientos arqueológicos o la ampliación del concepto de Patrimonio (abarcando desde elementos del Patrimonio Histórico y Etnográfico hasta el conjunto del paisaje como entorno construido culturalmente), potenciarían así el desarrollo, rentabilidad, efectividad y perspectivas de las actuaciones insertas en el ámbito de una Arqueología preventiva, como es el caso de los trabajos realizados en la fase de Evaluación de ImpactoArqueológico de la Red de Gasificación de Galicia.

En lo referente al plano organizativo, la práctica arqueológica desarrollada entre 1991 y 1997 generó una metodología que se adecúa al principal problema práctico generado por este tipo de proyectos, es decir, la unificación de criterios de las diferentes instancias implicadas en la Obra: laAdministración del Patrimonio Arqueológico, el equipo de arqueólogos y las empresas de ingeniería contratantes. Todo este trabajo ha sido un ejemplo positivo de incorporación de la Arqueología a un proyecto de obra pública y de colaboración entre la Empresa y la Universidad en aras de la protección del Patrimonio Arqueológico. Para ello fue necesario, no sólo, poner a punto metodologías de evaluación y criterios de corrección del Impacto Arqueológico, sino también establecer y consolidar una cultura de relación entre los arqueólogos y las empresas de

(14) En este punto es importante citar también el papel desempeñado por los Servicios de Arqueoloxía de la Consellería de Cultura como organismo ejecutor del imperativo legal de proteger el Patrimonio y controlar las acciones que intervienen sobre el mismo.

T. P., 58, n. $^{\circ} 1,2001$ 
ingeniería que, después de su inicial recelo hacia la Arqueología, crease una interacción basada en la confianza mutua y en la certeza de que las respectivas posiciones atendían a una orientación positiva cuyo fin último era beneficiar al proyecto reduciendo sus costes y haciéndolo viable. Esta tradición de colaboración, afianzada después de seis años de interrelación, constituye una de los principales bases para el trabajo arqueológico en obras públicas y podemos suponer que esta experiencia habrá servido así mismo a las empresas para familiarizarse con la problemática arqueológica y con sus formas de solución.

El planteamiento del trabajo arqueológico siguiendo un criterio homogéneo y el tratamiento uniforme de la información han permitido a su vez evaluar globalmente el impacto arqueológico de todos los proyectos que integran la Red de Gasificación de Galicia. Este método no sólo beneficia una correcta protección y gestión del Patrimonio Arqueológico afectado por las obras sino que contribuye a integrar todos los resultados generados por los trabajos de Evaluación de ImpactoArqueológico.

\section{CONSIDERACIONES FINALES}

El panorama que presenta la disciplina arqueológica en la actualidad en el Estado Español se define fundamentalmente por el divorcio (15) existente entre la práctica investigadora (Arqueología de Investigación) y la nueva orientación patrimonial (Arqueología de Gestión) surgida en las últimas décadas como consecuencia del desarrollo de políticas de Protección y Gestión del Patrimonio. La ausencia de propuestas globales que intentasen rentabilizar este carácter bidimensional de laArqueología ha permitido que el distanciamiento entre estas dos facetas desemboque muchas veces en un verdadero conflicto dentro del ámbito profesional y académico. Creemos que esta situación ha sido propiciada por la percepción de ambas como dos actividades antagónicas, partiendo del presupuesto de que responden a objetivos, estrategias metodológicas y problemáticas radicalmente distintas: mientras la denominada Arqueología de Investigación encarnaba la verdadera esencia y razón de ser de la disciplina, la Arqueología de Gestión era considerada como una mera técnica al servicio de los

(15) Un buen análisis de este proceso de distanciamiento puede verse en Criado (1996a) y Ruiz et alii (1999). intereses del mercado. De este modo, hemos visto cómo a lo largo de la última década se ha puesto en entredicho, de manera sistemática por parte de un amplio sector de la investigación, la rentabilidad y capacidad para generar conocimiento arqueológico de proyectos centrados en la protección del $\mathrm{Pa}$ trimonio.

El análisis global de los resultados obtenidos pone de manifiesto que la Evaluación de Impacto Arqueológico de la Red de Gasificación gallega no sólo constituye una contribución de primera magnitud a la protección y defensa del Patrimonio Cultural de Galicia, sino también un ejemplo de proyecto en el que se ha sabido conciliar la orientación esencialmente patrimonial del mismo con las exigencias propias de un programa de investigación. Hemos visto cómo la fase de Evaluación de Impacto Arqueológico ha desarrollado el estudio e interpretación del registro arqueológico documentado en la prospección superficial (16), así como una investigación aplicada orientada a la sistematización de metodologías y estrategias organizativas específicas para abordar la problemática impuesta por la gestión del Patrimonio Arqueológico. Esta rentabilización se alcanza adoptando una actitud que, sin justificar nunca una agresión patrimonial, se plantee como objetivo inmediato la formulación de una Arqueología que compense ese impacto, a veces inevitable, con la generación de conocimiento.

Por otro lado, el desarrollo de proyectos de intervención sobre el Patrimonio Arqueológico desde el ámbito universitario, como es el caso que nos ocupa, ha actuado como un incentivo crucial para la potenciación de la investigación arqueológica aplicada en la década de 1990 en nuestro país. Hay que tener en cuenta que durante los años 80 el control arqueológico de Obras Públicas era prácticamente inexistente, ya que se limitaba, en el mejor de los casos, a registrar en un informe los elementos arqueológicos destruidos por la obra de turno cuya afección directa sobre el Patrimonio era poco menos que considerada como inevitable.

De una Arqueología de Urgencia en ciernes, centrada en la consideración de los yacimientos de naturaleza visible y monumental catalogados en el área atravesada por el trazado, se ha pasado, por lo tanto, a estrategias globales y metodologías espe-

(16) Dicho registro nutre diversos trabajos de investigación básica actualmente en curso y ha servido, como se ha dicho anteriormente, de punto de partida para la realización de varias memorias de licenciatura y trabajos de investigación de Tercer Ciclo leídos en la Universidade de Santiago de Compostela. 
cíficas de Evaluación y Corrección de Impacto. Este avance trascendental, a nivel gallego y estatal (17), se debe en gran medida a la experiencia aportada por los trabajos de Evaluación de ImpactoArqueológico llevados a cabo por el GIArPa desde 1991, facilitados en gran medida por la legislación autonómica en materia de Protección del Patrimonio.

Como valoración final, cabe destacar que los trabajos de Evaluación de ImpactoArqueológico de la Red de Gasificación realizados entre 1991 y 1997, además de suponer un salto cualitativo en la consolidación de una Arqueología Preventiva en Galicia, así como un avance sustancial en la evaluación y minimización del efecto de este tipo de obras sobre los recursos culturales, sirven de punto de partida para valorar los bienes afectados, rentabilizar la información obtenida y profundizar en el conocimiento sobre el pasado de nuestro país.

\section{BIBLIOGRAFÍA}

Amado Reino, X.; Barreiro Martínez, D. y Martínez LÓPEZ, M. ${ }^{a}$ C. (1998): "Evaluación y corrección de impacto arqueológico en obras públicas. Propuestas desde la Arqueología del Paisaje". Arqueología Espacial, 1920: 153-64.

Amado Reino, X.; Martínez López, M. ${ }^{a}$ C. y Chao Álvarez, F.J. (1998): La Arqueología en la Gasificación de Galicia 2: Estudios de Evaluación de Impacto Arqueológico de la Red de Vigo-Porriño. TAPA, 4. Grupo de Investigación en Arqueoloxía da Paisaxe. Santiago.

Ayán Vila, J.M. y Amado Reino, X. (1999): La Arqueología en la Gasificación de Galicia 6: Estudios de Evaluación de Impacto. TAPA, 8. Grupo de Investigación en Arqueoloxía da Paisaxe. Santiago.

Barreiro Martínez, D. y Villoch Vázquez, V. (1997): Un Modelo de Evaluación de Impacto Arqueológico: El Parque Eólico de Careón. CAPA, 1. Grupo de Investigación en Arqueoloxía da Paisaxe. Santiago.

Catherall, P.D.; Barnett, M. y McClean, H. (eds.) (1984): The Southern Feeder. The Archaeology of a Gas Pipeline. The British Gas Corporation. London.

Cleary, R.M.; Hurley, M.F. y Twohig, E.A. (eds.) (1984): Archaeological excavations on the Cork-Dublin Gas

(17) Como ya hemos indicado, el proyecto de Control Arqueológico de Obras Públicas realizado por GIArPa fue el primero de estas características en el Estado Español. Actualmente se están empezando a conocer diferentes programas arqueológicos de evaluación y seguimiento de obras públicas, véanse por ejemplo los trabajos presentados en el $X X V$ Congreso Nacional de Arqueología celebrado en 1999 en Valencia, agrupados en un bloque temático específico (Arqueología de Seguimiento) que nos indica la importancia que ha alcanzado esta práctica arqueológica en la actualidad (Ruiz et alii, 1999; Miret y Mirò, 1999; Moreno y Bueno, 1999).

T. P., 58, n. ${ }^{\circ} 1,2001$
Pipeline (1981-1982). Cork Archaeological Studies, 1. Cork.

Cobas Fernández, I.; Criado Boado, F. y Prieto Martínez, M.'P. (1998): "Espacios del estilo: formas de la cultura material cerámica Prehistórica y Protohistórica en Galicia". Arqueología del Paisaje (Teruel, 1998). Arqueología Espacial, 19-20: 597-607.

Cobas Fernández, I. y Prieto Martínez, Ma.P. (1999): Introducción a la cerámica Prehistórica y Protohistórica en Galicia. TAPA, 17. Grupo de Investigación en Arqueoloxía da Paisaxe. Santiago.

CoDEX SCCL (1992): "Excavacions a l'autopista A-19, variant de Mataró. Tres exemples de poblament del Maresme: de l'ibèric ple a la romanització". Laietania, 7 : 157-189.

CONESA FERnÁNDEZ-VítoRA, V. (1995): Guía metodológica para la Evaluación de Impacto Ambiental. Ediciones Mundi-Prensa. Bilbao.

Criado Boado, F. (1989): "Resultados de la prospección intensiva en la Sierra de O Bocelo y en el valle del río Furelos (A Coruña)". Cuadernos de Estudios Gallegos, 103: 25-50.

- (1995): "El control arqueológico de obras de trazado lineal: planteamientos desde la arqueología del paisaje". Actas del XXII CNA (Vigo, 1993): 253-259. Vigo.

- (1996a): "La Arqueología del futuro, ¿el Futuro de la Arqueología?". Trabajos de Prehistoria, 53 (1): 15-36.

- (1996b). "La arqueología del paisaje como programa de gestión integral del patrimonio arqueológico". $\mathrm{Bo}$ letín del Instituto Andaluz del Patrimonio Histórico, 14: 15-19.

- $\quad$ (1996c): "Hacia un modelo integrado de investigación y gestión del Patrimonio Histórico: la cadena interpretativa como propuesta”. Boletín del Instituto Andaluz del Patrimonio Histórico, 16: 73-79.

- (1999): Del Terreno al Espacio: Planteamientos y Perspectivas para la Arqueología del Paisaje. CAPA, 6. Grupo de Investigación en Arqueoloxía da Paisaxe. Santiago.

Criado Boado, F.; Amado Reino, X. y Martínez López, $M^{a}$.C. (1997): "Red de Gasificación de Galicia. Corrección del Impacto Arqueológico". Revista de Arqueología, 198: 6-13.

- (1998): La Arqueología de la Gasificación de Galicia I: Plan de Control y Corrección de ImpactoArqueológico. CAPA, 4. Grupo de Investigación en Arqueoloxía da Paisaxe. Santiago.

Criado Boado, F.; Amado Reino, X.; Martínez López, M. ${ }^{a}$ C.; Cobas Fernández, I. y Parcero Oubiña, C. (2000): "Programa de Corrección del ImpactoArqueológico de la Gasificación de Galicia: un ejemplo de gestión integral del Patrimonio Arqueológico". Complutum, 11: 63-85.

Criado Boado, F.; Bonilla Rodríguez, A.; Cerqueiro landín, D.; GonzÁlez MÉndez, M.; Méndez Fernández, F. y Penedo Romero, R. (1988): "Proyecto Bocelo-Fure- 
los: arqueología del paisaje y prospección intensiva en Galicia". Trabalhos de Antropologia e Etnologia, 28: 241-250.

Criado Boado, F. (dir.); Bonilla Rodríguez, A.; Cerqueiro Landín, D.; Díaz VázQuez, M.; González MÉndez, M.; Infante Roura, F.; MÉndez Fernández, F.; Penedo Romero, R.; Rodríguez Puentes, E. y Vaquero LAstres, J. (1992): La Arqueología del Paisaje en Galicia. El área Bocelo-Furelos entre los tiempos paleolíticos y medievales. (Campañas de 1987, 1988 y 1989). Arqueoloxía/Investigación, 6. Dirección Xeral do Patrimonio Histórico. Xunta de Galicia. Santiago.

Criado Boado, F. y Parcero Oubiña, c. (1996): “Arqueología de las formas de parcelación del espacio en la prehistoria de Galicia”. En L. Guitián Rivera y R. Lois González (coords.): Actividad humana y cambios recientes en el paisaje. Consellería de Cultura. Xunta de Galicia. Santiago

Criado Boado, F.; Parcero Oubiña, C. y Villoch Vázquez, V. (1995): "Control arqueológico del oleoducto Coruña-Vigo. Fase II: seguimiento de las obras de construcción". Actas del XXII CNA (Vigo, 1993): 309-316.Vigo.

GómEz OrEA, D. (1994): Evaluación de ImpactoAmbiental. Editorial Agrícola Española, S.A. Madrid.

González Méndez, M.; Amado Reino, X. y Prieto MartíNEZ, P. (1995): "Control arqueológico de obras de trazado lineal: Trabajos previos de la Gasificación de Galicia”. Actas del XXII CNA (Vigo, 1993): 297-303. Vigo.

Lima Oliveira, E. y Prieto Martínez, M.P. (1999): La Arqueología de la Gasificación de Galicia 12: Intervenciones en yacimientos de la Prehistoria Reciente. TAPA, 16. Grupo de Investigación en Arqueoloxía da Paisaxe. Santiago.

MAGGI, R.(1992): Archeologia preventiva lungo il precorso di un metanodotto. Il tratto Genova-derivazione per Recco. Quaderni della Soprintendenza Archeologica della Liguria, 4. Génova.

Martínez López, M. ${ }^{\mathrm{a} C}$.; Amado Reino, X. y Barreiro MARTÍNEZ, D. (1997): “Managing Archaeological impact: from Evaluation to Correction”. TAPA, 2. Grupo de Investigación enArqueoloxía da Paisaxe. Santiago de Compostela: 41-46.

MÉndez Fernández, F. (1994): "La domesticación del paisaje durante la Edad del Bronce gallego". Trabajos de Prehistoria, 51(1): 77-94.

- (1998): "Definición y análisis de poblados de la Edad del Bronce en Galicia”. En R. Fábregas (ed.): A Idade do Bronce en Galicia: novas perspectivas. Ediciós do Castro. A Coruña: 153-189.

Méndez Fernández, F.; GonzÁlez Méndez, M. y Amado Reino, X. (1995): “Control Arqueológico del Oleoducto Coruña-Vigo. Fase I: Trabajos Previos y Superficiales". Actas del XXII CNA (Vigo, 1993): 293-296. Vigo.
Méndez Fernández, F.; Prieto Martínez, P. y Villoch VÁzquEz, V. (1995): "Evaluación del ImpactoArqueológico de las Autovías del M.O.P.T. (Lugo-A Coruña y Ourense-Porriño)". Actas del XXII CNA (Vigo, 1993): 305-308. Vigo.

Miret Mestre, M. y Miró Alaix, M. aT. (1999). “Intervenciones arqueológicas en el tramo Sitges/ElVendrell de la Autopista Pau CasalsA-16 (Barcelona/Comaruga)". Actas del XXV Congreso Nacional de Arqueología (València, 1999): 420-424. València.

Moreno Pelayo, R.M. y Bueno Moreno, M. (1999): “Seguimiento arqueológico de los gasoductos Tarifa-Córdoba (Tramos I y II), Córdoba-Frontera con Portugal (Tramo I) y Zamora-Benavente-León. Aplicación de metodología para preservación de yacimientos arqueológicos en grandes obras civiles". Actas del XXV Congreso Nacional de Arqueología (València, 1999): 428430. València.

PARCERo Oubiña, C. (1997): Documentación de un Entorno Castreño: Trabajos Arqueológicos en el área de Cameixa, Ourense. TAPA, 1. Grupo de Investigación en Arqueoloxíą da Paisaxe. Santiago.

- (1998): La Arqueología en la Gasificación de Galicia 3: Excavación del Túmulo $n^{\circ} 3$ delAlto de San Cosme. TAPA, 5. Grupo de Investigación en Arqueoloxía da Paisaxe. Santiago.

- (1999): La Arqueología en la Gasificación de Galicia 7: Hacia unaArqueología Agraria de la Cultura Castreña. TAPA, 8. Grupo de Investigación en Arqueoloxía da Paisaxe. Santiago.

Ruiz, A.; Zafra, N.; Hornos, F. y Castro, M. (1999): “El Seguimiento de la intervención arqueológica: el caso de Marroquíes Bajos en Jaén". Actas del XXV Congreso Nacional de Arqueología (València, 1999): 407-419. València.

SAntos Estévez, M. (1998): "Los espacios del arte: el diseño del panel y articulación del paisaje en el arte rupestre gallego". Trabajos de Prehistoria, 55 (2): 73-88.

Santos Estévez, M. y Criado Boado, F. (1998): "Espacios rupestres: del panel al paisaje". Arqueología del Paisaje (Teruel, 1998). Arqueología Espacial, 19-20: 579595.

SuÁrez Cardona, F. (1991): Guías Metodológicas para la elaboración de estudios de impacto ambiental. Monografías de la Secretaría de Estado para las Políticas del Agua y el MedioAmbiente 1. Carreteras y Ferrocarriles. MOPT. Madrid, $2^{\mathrm{a}}$ edición.

VILLOCH VÁzQUEZ, V. (1995): “Monumentos y petroglifos: la construcción del espacio en las sociedades constructoras de túmulos del noroeste peninsular". Trabajos de Prehistoria, 52 (1): 39-55.

Villoch Vázquez, V. y Barreiro Martínez, D. (1997): “AArqueología vs. Energía eólica?”. Boletín del Instituto Andaluz del Patrimonio Histórico, 20: 103-108. 